\title{
A Best Technique of Weight Lose using Fuzzy Soft Systems
}

\author{
Muhammad Naveed Jafar \\ Department of Mathematics Lahore \\ Garrison University \\ Sector-C, DHA Phase-VI 54000, \\ Lahore Pakistan
}

\author{
Ali Hamza \\ Department of Mathematics Lahore \\ Garrison University \\ Sector-C, DHA Phase-VI 54000, \\ Lahore Pakistan
}

\author{
Sara Farooq \\ Department of Mathematics Lahore \\ Garrison University \\ Sector-C, DHA Phase-VI 54000, \\ Lahore Pakistan
}

\begin{abstract}
Soft set become very handy tool to solve decision making problems of daily life and Fuzzy Hyper soft set is the generalized foam of soft set. In this current approach, a systematic, logical and consistent use of Fuzzy hyper soft set theory, which include from estimate of ideal weight to time required for this transformation.
\end{abstract}

\section{Keywords}

Fuzzy Soft Systems

\section{INTRODUCTION}

Currently, the concept of Multi Criteria Decision Making and fuzzy set is widely used in different fields world-wide [1-3] [14] [23], as well as these concept are using in medication and selection of things in daily life [4-8]. As well as fitness is a serious matter on which we should think studiously. Fitness is major for survival. Abstraction of Fuzzy set become really convenient to work on, the concept of soft set was proposed by Molodtsov in 1999 [9] and further Maji.et.al proposed Intuitionistic fuzzy matrices [10].. Rough set theory developed by Z. Pawlak in 1982 [11-12]. The Extended COWG operators [13] also plays an important role in this current era of research. We studied a fair amount of statistics and in this current epoch we use this all criteria and put the concept of Fuzzy on it. According to a survey, in America only $16 \%$ Americans belong to health clubs. Over daily life become really luxurious life so the diseases rise, like diabetes, blood pressure, increase of cholesterol and the most common disease Obesity which percentage in America is $39.8 \%$. According to a survey, almost 2.8 million adults die due to obesity or overweight every year. Obesity is also a reason of heart disease and as well as Cancer. Obesity is the fifth cause of death, as compare to males, females more suffers from obesity. Let us mention that regain of weight after weight loss is common which depends upon your behavior, diet, and environment. People usually do the exercise between the ages of 18 to 24 world-wide. Only $20.6 \%$ people fulfill the requirement of exercise of our body. As compare to female, male loss weight rapidly. In year 1995, Smarandache [15] proposed a new concept and Neutrosophic sets firstly given by Smarandache in in 1998 [16-17]. Researches from different zone currently work on multi-criteria decision making and proposed some new results [18-22]. Fuzzy set proposed by Lotfi Zadeh [24] in 1965 and Algorithm for priority of fuzzy complementary judgment matrix", also put these concepts forward [25].

Now, we will put light on Diet. You just have to increase the consumption of vegetables and fruits, decrease the level of carbohydrates such as rice and bread and increase the level of protein by using meat. Increase the daily usage of water and decrease the usage of salt and spices. Low fat diet help to increase the metabolism, here metabolism plays a very important role in weight loss. If we used to take meal with high glycemic, increases the ratio of insulin to glucagon, it held the body on storage the food as compare to the process of oxidation. In other word high conception of carbohydrates held to obesity because it is easy to digest and over body try to store as more as it can. On the other-hand protein and other minerals and vitamins from vegetables, meat and fruit are beneficial for metabolism and help us to loss fat. All type of fast food and soft drinks are foe of health. The selection of ground for walk, jogging and running is also essential for this journey, the track should be suitable like dirt and synthetic track to keep injures away. In this epoch, we make an organized setup which help you to identify, how much you have to loss, the time and progress as well as the effects are mentioned.

The complete low-fat diet found by different studies. The DASH $\dot{H}$ eating pattern also available which is really effective diet for weight loss, sponsored by NHLBI. The DAS $\dot{H}$ diet consist of increased protein intake with more fruits and vegetables usage. According to serval researches in 2006 obesity cause $\$ 40$ billion spending in medical. Further it was about $\$ 147$ billion in 2008 , for DAS $\dot{H}$ the intakes of carbohydrates is about $53.3 \%$ in first phase and $51 \%$ in the second phase. The regain of weight is also common, it is proved by researchers that cardiovascular risk factor improved by modest weight loss.

\subsection{Motivation}

In this study, we collect the data and statistics from different universities and statistics from world-wide, to estimate the time required to weight loss by the help of fuzzy set as well as our contribution is to make a organized structure, which gave a complete guide to understand the phenomena and after applying it, you will get a result. Obesity is a serious issue of current life style, so by analysis this setup you should be able to get a complete idea.

\section{STRUCTURE OF THIS PAPER}

In this current research article section 1 contains Introduction and Motivation, section 2 contains definitions and numerical problem to understand Fuzzy hyper soft set, section 3 depends upon parameter value and Neutrosophic fuzzy values of different exercises, In section 4 Identification of target (healthy weight by height), selection of target in section 5 and numerical example in section 6.

\subsection{Soft Set}

Let $\boldsymbol{U}$ is universal initial set and $\dddot{\mathrm{E}}$ is parameter set. Let $\dot{p}(\boldsymbol{U})$ donates as power set of $\dot{U}$ and $\dot{\mathcal{A}} \subseteq \mathbb{E}$. We can say pair $(\dot{\mathcal{F}}, \mathbb{A})$ 
is sot set over $\dot{\mathbb{U}}$, where $\dot{\mathcal{F}}$ shows the mapping given by $\dot{\mathcal{F}}: \rightarrow \dot{p}$ $(\boldsymbol{U}) .(\dot{F}, \mathbb{A})$ given as

$(\dot{F}, \mathbb{A})=\{\dot{F}($ e $) \in \mathbb{P}(\boldsymbol{U}):$ e $\in \boldsymbol{E}, \dddot{F}(\mathrm{e})=\varnothing$ if e $\nsubseteq \ddot{\mathcal{A}}\}$

\subsection{Hyper Soft Set}

Here we have the universal set as $\boldsymbol{u}$, power set is defined as (ㄸ). As well as $\mathbb{a}_{1}, \mathbb{a}_{2}, \mathbb{a}_{3}, \ldots, \mathbb{a}_{n}$ here $\dot{n} \geq 1$, be well defined until $\mathrm{n}$. with the corresponding value of attributes as value of attributes as $\mathcal{A}_{1}, \mathcal{A}_{2}, \mathcal{A}_{3}, \ldots, \mathcal{A}_{n}$ with $\mathcal{A}_{i} \cap \mathcal{A}_{j}=\phi$ for $i \neq j$ as well as $i, \boldsymbol{j}=(1,2, \ldots, \dot{n})$ then we get the pair as $\left(\mathcal{F}, \mathcal{A}_{1}, \mathcal{A}_{2}, \mathcal{A}_{3}, \ldots, \mathcal{A}_{n}\right), \quad$ and $\quad \mathcal{F}, \mathcal{A}_{1}, \mathcal{A}_{2}, \mathcal{A}_{3}, \ldots, \mathcal{A}_{n} \longmapsto$ $\mathcal{P}$ (uㅏ).

\section{Numerical Problem}

Here $\mathcal{U}=\left\{\mathbb{x}_{1}, \mathbb{x}_{2}, \mathbb{x}_{3}, \mathbb{x}_{4}\right\}$ and a set $\mathcal{N}=\left\{\mathcal{F}_{1}, \mathcal{F}_{2}, \mathcal{F}_{3}, \mathcal{F}_{5}\right\} \subset \mathcal{U}$.

Let the attribute $\mathbb{a}_{1}=$ walk, $\mathbb{a}_{2}=$ jogging, $\mathbb{a}_{3}=$ running, $\mathrm{a}_{4}=\mathrm{jim}$, now the attributes values are:

Walk $=\mathcal{F}_{1}=\left\{\overline{\bar{h}}_{1}, \overline{\bar{h}}_{2}, \overline{\bar{h}}_{3}, \overline{\bar{h}}_{4}, \overline{\bar{h}}_{5}\right\}, \quad$ Yoga

stretching $=\mathcal{F}_{2}=\left\{\overline{\bar{h}}_{1}, \overline{\bar{h}}_{2}, \overline{\bar{h}}_{3}, \overline{\bar{h}}_{4}, \overline{\bar{h}}_{5}\right\}$

Jogging/cycling $=\mathcal{F}_{3}=\left\{\overline{\bar{h}}_{1}, \overline{\bar{h}}_{2}, h_{3}, \overline{\bar{h}}_{4}, h_{5}\right\}$, Running / Split $=\mathcal{F}_{4}=\left\{h_{1}, h_{2}, \overline{\bar{h}}_{3}, h_{4}, \overline{\bar{h}}_{5}\right\}$
Sports $=\mathcal{F}_{5}=\left\{\tilde{\bar{h}}_{1}, \overline{\bar{h}}_{2}, h_{3}, \overline{\bar{h}}_{4}, h_{5}\right\}$. Then the function will be $\mathcal{F}: \mathcal{A}_{1} \times \mathcal{A}_{2} \times \mathcal{A}_{3} \times \mathcal{A}_{4} \mapsto$ (nn)

Let's assume that $\{\overleftrightarrow{(\phi)}\}=\left\{\mathcal{F}_{1}, \mathcal{F}_{2}, \mathcal{F}_{3}, \mathcal{F}_{5}\right\}$ with respect to the set $\mathcal{N}$.

\subsection{Neutrosophic Set}

A Universe of Discourse $\mathcal{U}_{\mathcal{N}}$ is Neutrosophic if $\forall \mathcal{F} \in \mathcal{U}_{\mathcal{N}}, \mathcal{F}$ partially belongs, partially do not belong and some hesitation value called indeterminacy. Let's denote as $\left(\mathcal{J}_{\mathcal{F}}, \mathcal{J}_{\mathcal{F}}, \mathcal{F}_{\mathcal{F}}\right)$.

\section{NEUTROSOPHIC FUZZY VALUES OF EXERCISES AND PARAMETER FOR SOFT RULES}

In this table, each value consist of three different parameters such as truthiness, falsity and indeterminacy. Now consider walk, for weight loss the walk required with full effectiveness because our body is used to of it. Walk are slow process for weight loss and some time it become non-beneficial so the value for walk according this table is 0.6 for truthiness, 0.4 for falsity and 0.2 for indeterminacy.

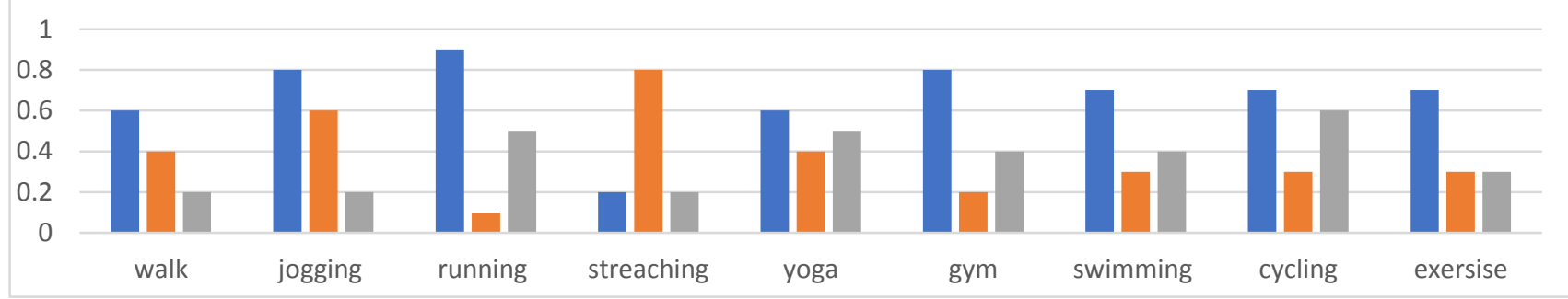

Figure.01

\begin{tabular}{|c|c|c|c|c|c|c|c|c|c|c|c|c|c|c|c|c|c|c|c|c|c|c|c|c|}
\hline LBS & 100 & 105 & 110 & 115 & 120 & 125 & 130 & 135 & 140 & 145 & 150 & 155 & 160 & 165 & 170 & 175 & 180 & 185 & 190 & 195 & 200 & 205 & 210 & 215 \\
\hline KGS & 45.5 & 47.5 & 50.0 & 52.3 & 54.5 & 55.8 & 59.1 & 61.4 & 63.6 & 65.9 & 68.2 & 70.5 & 72.7 & 75.0 & 77.3 & 79.5 & 81.8 & 84.1 & 86.4 & 88.6 & 90.9 & 93.2 & 95.5 & 97.7 \\
\hline $5^{\prime} 0$ & 19 & 20 & 21 & 22 & 23 & 24 & 25 & 26 & 27 & 28 & 29 & 30 & 31 & 32 & 33 & 34 & 35 & 36 & 37 & 38 & 39 & 40 & 41 & 42 \\
\hline $5 ' 1$ & 18 & 19 & 20 & 21 & 22 & 23 & 24 & 25 & 26 & 27 & 28 & 29 & 30 & 31 & 32 & 33 & 34 & 35 & 36 & 36 & 37 & 38 & 39 & 40 \\
\hline $5 ’ 2$ & 18 & 19 & 20 & 21 & 22 & 22 & 23 & 24 & 25 & 26 & 27 & 28 & 29 & 30 & 31 & 32 & 33 & 33 & 34 & 35 & 36 & 37 & 38 & 39 \\
\hline 5,3 & 17 & 18 & 19 & 20 & 21 & 22 & 23 & 24 & 24 & 25 & 26 & 27 & 28 & 29 & 30 & 31 & 32 & 32 & 33 & 34 & 35 & 36 & 37 & 38 \\
\hline 5,4 & 17 & 18 & 18 & 19 & 20 & 21 & 22 & 23 & 24 & 24 & 25 & 26 & 27 & 28 & 29 & 30 & 31 & 31 & 32 & 33 & 34 & 35 & 36 & 37 \\
\hline 5,5 & 16 & 17 & 18 & 19 & 20 & 20 & 21 & 22 & 23 & 24 & 25 & 25 & 26 & 27 & 28 & 29 & 30 & 30 & 31 & 32 & 33 & 34 & 35 & 35 \\
\hline $5 \% 6$ & 16 & 17 & 17 & 18 & 19 & 20 & 21 & 21 & 22 & 23 & 24 & 25 & 25 & 26 & 27 & 28 & 29 & 29 & 30 & 31 & 32 & 33 & 34 & 34 \\
\hline $5 ' 7$ & 15 & 16 & 17 & 18 & 18 & 19 & 20 & 21 & 22 & 22 & 23 & 24 & 25 & 25 & 26 & 27 & 28 & 29 & 29 & 30 & 31 & 32 & 33 & 33 \\
\hline 5,8 & 15 & 16 & 16 & 17 & 18 & 19 & 19 & 20 & 21 & 22 & 22 & 23 & 24 & 25 & 25 & 26 & 27 & 28 & 28 & 29 & 30 & 31 & 32 & 32 \\
\hline 599 & 14 & 15 & 16 & 17 & 17 & 18 & 19 & 20 & 20 & 21 & 22 & 22 & 23 & 24 & 25 & 25 & 26 & 27 & 28 & 28 & 29 & 30 & 31 & 31 \\
\hline $5^{\prime} 10$ & 14 & 15 & 15 & 16 & 17 & 18 & 18 & 19 & 20 & 20 & 21 & 22 & 22 & 23 & 24 & 25 & 25 & 26 & 27 & 28 & 28 & 29 & 30 & 30 \\
\hline 5'11 & 14 & 14 & 15 & 16 & 16 & 17 & 18 & 18 & 19 & 20 & 21 & 21 & 22 & 23 & 23 & 24 & 25 & 25 & 26 & 27 & 27 & 28 & 29 & 30 \\
\hline $6^{\prime} 0$ & 13 & 14 & 14 & 15 & 16 & 17 & 17 & 18 & 19 & 19 & 20 & 21 & 21 & 22 & 23 & 23 & 24 & 25 & 25 & 26 & 27 & 27 & 28 & 29 \\
\hline 6'1 & 13 & 13 & 14 & 15 & 15 & 16 & 17 & 17 & 18 & 19 & 19 & 20 & 21 & 21 & 22 & 23 & 23 & 24 & 25 & 25 & 26 & 27 & 27 & 28 \\
\hline 622 & 12 & 13 & 14 & 14 & 15 & 16 & 16 & 17 & 18 & 18 & 19 & 19 & 20 & 21 & 21 & 22 & 23 & 23 & 24 & 25 & 25 & 26 & 27 & 27 \\
\hline
\end{tabular}




\begin{tabular}{|c|c|c|c|c|c|c|c|c|c|c|c|c|c|c|c|c|c|c|c|c|c|c|c|c|}
\hline $6{ }^{\prime} 3$ & 12 & 13 & 13 & 14 & 15 & 15 & 16 & 16 & 17 & 18 & 18 & 19 & 20 & 20 & 21 & 21 & 22 & 23 & 23 & 24 & 25 & 25 & 26 & 26 \\
\hline $6 \% 4$ & 12 & 13 & 13 & 14 & 14 & 15 & 15 & 16 & 17 & 17 & 18 & 18 & 19 & 20 & 20 & 21 & 22 & 22 & 23 & 23 & 24 & 25 & 25 & 26 \\
\hline
\end{tabular}

Table.01 and 02 to estimate the weight with respect to health

\begin{tabular}{|c|c|}
\hline Time & Trait \\
\hline $\mathbf{1 5} \mathbf{~ m i n}$ & 0.25 \\
\hline $\mathbf{3 0} \mathbf{~ m i n}$ & 0.5 \\
\hline $\mathbf{4 5} \mathbf{~ m i n}$ & 0.75 \\
\hline $\mathbf{1}$ hour & 1 \\
\hline
\end{tabular}

\section{4-a) Identification of target}

First we will estimate how much a person need to loss for this we a table is given below. This current table have a complete guide on how much a person need to loss this table consist of

\section{4-b) Selection of target}

Now, by using these statistics and soft rules we will be able to start the journey of weight loss. height and weight, different levels are mentioned but the main one is obesity e.g. for the person with height 5 ' 10 from $59 \mathrm{~kg}$ to $77 \mathrm{~kg}$ is the healthy weight and the weight from $77 \mathrm{~kg}$ to onward is over-weight.

Table 03

\begin{tabular}{|c|c|c|c|c|c|c|}
\hline $\begin{array}{l}\text { LBS } \\
\text { KGS }\end{array}$ & $\begin{array}{l}134-138 \\
61-62.9\end{array}$ & $\begin{array}{l}138-143 \\
63-64.9\end{array}$ & $\begin{array}{l}143-147 \\
65-66.9\end{array}$ & $\begin{array}{l}147-152 \\
67-68.9\end{array}$ & $\begin{array}{l}152-156 \\
69-70.9\end{array}$ & $\begin{array}{l}156-160 \\
71-72.9\end{array}$ \\
\hline Category & $\overleftrightarrow{A}$ & $\overleftrightarrow{B}$ & $\overleftrightarrow{C}$ & $\overleftrightarrow{D}$ & $\overleftrightarrow{E}$ & $\overleftrightarrow{F}$ \\
\hline LBS & $160-165$ & $165-169$ & $169-174$ & $174-178$ & $178-182$ & $182-187$ \\
\hline KGS & 73-74.9 & $75-76.9$ & 77-78.9 & 79-80.9 & $81-82.9$ & 83-84.9 \\
\hline Category & $\overleftrightarrow{G}$ & $\overleftrightarrow{H}$ & $\overleftrightarrow{I}$ & $\overleftrightarrow{J}$ & $\overleftrightarrow{K}$ & $\overleftrightarrow{L}$ \\
\hline LBS & $187-191$ & 191-196 & $196-200$ & $200-205$ & $205-209$ & $209-213$ \\
\hline KGS & $85-86.9$ & $87-88.9$ & 89-90.9 & $91-92.9$ & 93-94.9 & $95-96.9$ \\
\hline Category & $\overleftrightarrow{M}$ & $\overleftrightarrow{N}$ & $\overleftrightarrow{O}$ & $\overleftrightarrow{P}$ & $\overleftrightarrow{Q}$ & $\overleftrightarrow{R}$ \\
\hline LBS & $213-218$ & $218-222$ & $222-227$ & $227-231$ & $231-235$ & $235-240$ \\
\hline KGS & $97-98.9$ & 99-100.9 & $101-102.9$ & $103-104.9$ & $105-106.9$ & $107-108.9$ \\
\hline Category & $\overleftrightarrow{S}$ & $\overleftrightarrow{T}$ & $\overleftrightarrow{U}$ & $\overleftrightarrow{V}$ & $\overleftrightarrow{W}$ & $\overleftrightarrow{X}$ \\
\hline LBS & $240-244$ & $244-249$ & $249-253$ & $253-257$ & $257-262$ & $262-266$ \\
\hline KGS & $109-110.9$ & $111-112.9$ & $113-114.9$ & $115-116.9$ & $117-118.9$ & $119-120.9$ \\
\hline Category & $\overleftrightarrow{Y}$ & $\overleftrightarrow{Z}$ & $\overleftrightarrow{\Omega}$ & $\overleftrightarrow{\mathrm{?}}$ & $\overleftrightarrow{\varepsilon}$ & $\overleftrightarrow{v}$ \\
\hline LBS & $266-271$ & $271-275$ & $275-279$ & $279-284$ & $284-288$ & $288-293$ \\
\hline KGS & $121-122.9$ & $123-124.9$ & $125-126.9$ & $127-128.9$ & $129-130.9$ & $131-133$ \\
\hline Category & $\overleftrightarrow{\text { ? }}$ & $\overleftrightarrow{\mathrm{o}}$ & $\overleftrightarrow{\psi}$ & $\overleftrightarrow{\phi}$ & $\overleftrightarrow{\partial}$ & $\overleftrightarrow{\xi}$ \\
\hline
\end{tabular}

By this table we will select the category in which our weight lye, e.g. a person with $86.4 \mathrm{~kg}$ will select $\overleftrightarrow{M}$. Once u selected the category the procedure start. According to different surveys, documentaries and experiences, A person need 1 week to loss $2 \mathrm{kgs}$, so each category lye for 1 week. After it you will move forward in next category and so on.

\section{4-c )Soft Rules}

We take a start from extremely obese and the rules move towards healthy weight.

In early days make your body adaptive for this by simple fast walk and short distance jogging, running and basic exercises. 
Increase metabolism and consumption of water for better results.

$$
\begin{aligned}
& \mathcal{F}(\{\overleftrightarrow{\phi}\})= \\
& \left\{\mathcal{F}_{1}(0.5), \mathcal{F}_{2}(0.25), \mathcal{F}_{3}(0.25), \mathcal{F}_{4}(0), \mathcal{F}_{5}(0.5)\right\} \\
& \mathcal{F}\left(\left\{\overleftrightarrow{\xi\})}=\left\{\mathcal{F}_{1}(0.5), \mathcal{F}_{2}(0.25), \mathcal{F}_{3}(0.25), \mathcal{F}_{4}(0), \mathcal{F}_{5}(0.5)\right\}\right.\right. \\
& \mathcal{F}(\{\overleftrightarrow{\partial}\})=\left\{\mathcal{F}_{1}(0.5), \mathcal{F}_{2}(0.25), \mathcal{F}_{3}(0.25), \mathcal{F}_{4}(0), \mathcal{F}_{5}(0.5)\right\} \\
& \widetilde{\mathcal{F}(\{\phi}\})=\left\{\mathcal{F}_{1}(0.5), \mathcal{F}_{2}(0.25), \mathcal{F}_{3}(0.25), \mathcal{F}_{4}(0), \mathcal{F}_{5}(0.5)\right\} \\
& \mathcal{F}(\overleftrightarrow{\{\psi}\})=\left\{\mathcal{F}_{1}(0.5), \mathcal{F}_{2}(0.25), \mathcal{F}_{3}(0.25), \mathcal{F}_{4}(0), \mathcal{F}_{5}(0.5)\right\} \\
& \mathcal{F}(\{\dot{0}\})=\left\{\mathcal{F}_{1}(0.5), \mathcal{F}_{2}(0.25), \mathcal{F}_{3}(0.25), \mathcal{F}_{4}(0), \mathcal{F}_{5}(0.5)\right\}
\end{aligned}
$$

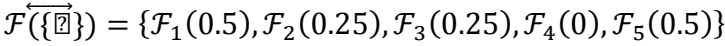

$$
\begin{aligned}
& \widetilde{\mathcal{F}(\{\mathrm{v}\})}=\left\{\mathcal{F}_{1}(0.25), \mathcal{F}_{2}(0.25), \mathcal{F}_{3}(0.5), \mathcal{F}_{4}(0), \mathcal{F}_{5}(0.5)\right\} \\
& \mathcal{F}(\{\xi\})=\left\{\mathcal{F}_{1}(0.25), \mathcal{F}_{2}(0.25), \mathcal{F}_{3}(0.5), \mathcal{F}_{4}(0), \mathcal{F}_{5}(0.5)\right\} \\
& \mathcal{F}(\{\})=\left\{\mathcal{F}_{1}(0.25), \mathcal{F}_{2}(0.25), \mathcal{F}_{3}(0.5), \mathcal{F}_{4}(0), \mathcal{F}_{5}(0.5)\right\}
\end{aligned}
$$

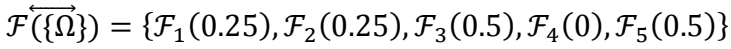

$$
\begin{aligned}
& \widetilde{\mathcal{F}(\{Z}\})=\left\{\mathcal{F}_{1}(0.25), \mathcal{F}_{2}(0.25), \mathcal{F}_{3}(0.5), \mathcal{F}_{4}(0), \mathcal{F}_{5}(0.5)\right\} \\
& \mathcal{F}(\{\overleftrightarrow{Y}\})=\left\{\mathcal{F}_{1}(0.25), \mathcal{F}_{2}(0.25), \mathcal{F}_{3}(0.5), \mathcal{F}_{4}(0), \mathcal{F}_{5}(0.5)\right\} \\
& \mathcal{F}(\{\overleftrightarrow{X}\})=\left\{\mathcal{F}_{1}(0.25), \mathcal{F}_{2}(0.25), \mathcal{F}_{3}(0.5), \mathcal{F}_{4}(0), \mathcal{F}_{5}(0.5)\right\} \\
& \overrightarrow{\mathcal{F}}(\{W\})=\left\{\mathcal{F}_{1}(0.25), \mathcal{F}_{2}(0.25), \mathcal{F}_{3}(0.5), \mathcal{F}_{4}(0), \mathcal{F}_{5}(0.5)\right\} \\
& \overrightarrow{\mathcal{F}(\{V\})}=\left\{\mathcal{F}_{1}(0.25), \mathcal{F}_{2}(0.25), \mathcal{F}_{3}(0.5), \mathcal{F}_{4}(0), \mathcal{F}_{5}(0.5)\right\} \\
& \mathcal{F}(\{U\})=\left\{\mathcal{F}_{1}(0.25), \mathcal{F}_{2}(0.25), \mathcal{F}_{3}(0.25), \mathcal{F}_{4}(0.25), \mathcal{F}_{5}(0.5)\right\} \\
& \mathcal{F}(\{T\})=\left\{\mathcal{F}_{1}(0.25), \mathcal{F}_{2}(0.25), \mathcal{F}_{3}(0.25), \mathcal{F}_{4}(0.25), \mathcal{F}_{5}(0.5)\right\}
\end{aligned}
$$

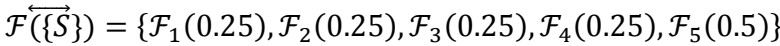

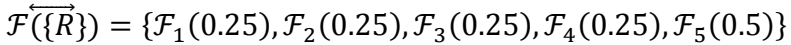

$$
\begin{aligned}
& \mathcal{F}(\overleftrightarrow{Q Q}\})=\left\{\mathcal{F}_{1}(0.25), \mathcal{F}_{2}(0.25), \mathcal{F}_{3}(0.25), \mathcal{F}_{4}(0.25), \mathcal{F}_{5}(0.5)\right\} \\
& \mathcal{F}(\{\overleftrightarrow{P}\})=\left\{\mathcal{F}_{1}(0.25), \mathcal{F}_{2}(0.25), \mathcal{F}_{3}(0.25), \mathcal{F}_{4}(0.25), \mathcal{F}_{5}(0.5)\right\} \\
& \overrightarrow{\mathcal{F}}(\{0\})=\left\{\mathcal{F}_{1}(0.25), \mathcal{F}_{2}(0.25), \mathcal{F}_{3}(0.25), \mathcal{F}_{4}(0.25), \mathcal{F}_{5}(0.5)\right\} \\
& \mathcal{F}(\{N\})=\left\{\mathcal{F}_{1}(0.25), \mathcal{F}_{2}(0.25), \mathcal{F}_{3}(0.25), \mathcal{F}_{4}(0.25), \mathcal{F}_{5}(0.5)\right\} \\
& \overrightarrow{\mathcal{F}(\{M}\})=\left\{\mathcal{F}_{1}(0.25), \mathcal{F}_{2}(0.25), \mathcal{F}_{3}(0.25), \mathcal{F}_{4}(0.25), \mathcal{F}_{5}(0.5)\right\} \\
& \widetilde{\mathcal{F}(\{L}\})=\left\{\mathcal{F}_{1}(0.25), \mathcal{F}_{2}(0), \mathcal{F}_{3}(0.5), \mathcal{F}_{4}(0.25), \mathcal{F}_{5}(0.5)\right\} \\
& \overrightarrow{\mathcal{F}(\{K\})}=\left\{\mathcal{F}_{1}(0.25), \mathcal{F}_{2}(0), \mathcal{F}_{3}(0.5), \mathcal{F}_{4}(0.25), \mathcal{F}_{5}(0.5)\right\}
\end{aligned}
$$

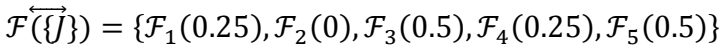

$$
\begin{aligned}
& \mathcal{F}(\{\overleftrightarrow{I}\})=\left\{\mathcal{F}_{1}(0.25), \mathcal{F}_{2}(0), \mathcal{F}_{3}(0.5), \mathcal{F}_{4}(0.25), \mathcal{F}_{5}(0.5)\right\} \\
& \mathcal{F}(\{\overleftrightarrow{H}\})=\left\{\mathcal{F}_{1}(0.25), \mathcal{F}_{2}(0), \mathcal{F}_{3}(0.5), \mathcal{F}_{4}(0.25), \mathcal{F}_{5}(0.5)\right\} \\
& \mathcal{F}(\{\overleftrightarrow{G}\})=\left\{\mathcal{F}_{1}(0.25), \mathcal{F}_{2}(0), \mathcal{F}_{3}(0.5), \mathcal{F}_{4}(0.25), \mathcal{F}_{5}(0.5)\right\}
\end{aligned}
$$

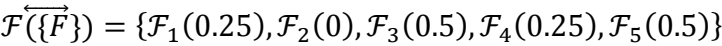

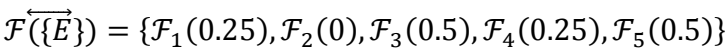

$$
\begin{aligned}
& \overleftrightarrow{\mathcal{F}(\{D}\})=\left\{\mathcal{F}_{1}(0), \mathcal{F}_{2}(0), \mathcal{F}_{3}(0.5), \mathcal{F}_{4}(0.25), \mathcal{F}_{5}(0.75)\right\} \\
& \mathcal{F}(\{\overleftrightarrow{C}\})=\left\{\mathcal{F}_{1}(0), \mathcal{F}_{2}(0), \mathcal{F}_{3}(0.5), \mathcal{F}_{4}(0.25), \mathcal{F}_{5}(0.75)\right\} \\
& \mathcal{F}(\{\overleftrightarrow{B}\})=\left\{\mathcal{F}_{1}(0), \mathcal{F}_{2}(0), \mathcal{F}_{3}(0.5), \mathcal{F}_{4}(02.5), \mathcal{F}_{5}(0.75)\right\} \\
& \mathcal{F}(\{\overleftrightarrow{A}\})=\left\{\mathcal{F}_{1}(0), \mathcal{F}_{2}(0), \mathcal{F}_{3}(0.5), \mathcal{F}_{4}(0.25), \mathcal{F}_{5}(0.75)\right\}
\end{aligned}
$$

These are the rules which can be use for weight loss.

\section{By multiply each rule by 7 we get the total days.}

Graphical representation;
Each rule are used to lose $2 \mathrm{kgs}$ and the time required is at least 5 days of work in a week for each one, so by multiplying these rules by 7 we can estimate the time required.

Some other rules for random people.

(a) For a person with fit body but he wants to increase his stamina and strength.

This person can follow different rule for short period of time and modify the rules according to his requirement.

(b) For a married person who wants to follow a single rule for healthy body.

$(F, \overleftrightarrow{\mathbb{I I}})=\left\{\mathcal{F}_{1}(0.25), \mathcal{F}_{2}(0.25), \mathcal{F}_{3}(0.5), \mathcal{F}_{4}(0), \mathcal{F}_{5}(0.5)\right\}$

\section{NUMERICAL PROBLEMS}

Mr. John have height 5'10 inch, has weight is $112 \mathrm{kgs} \mathrm{He}$ want to loss-weight.

Step 1. By using the table we get a result that for healthy life MR. John need to loss weight and put it less than 78 because the healthy weight for 5,10 from $59 \mathrm{~kg}$ to $77 \mathrm{~kg}$ is the healthy weight and the weight from $77 \mathrm{~kg}$ to onward is over-weight.

Step 2. Now Mr. john will select rules for weight loss, they become used to of walk and exercise and then the rules selected for Mr. John are;

From the journey of 112 to $77 \mathrm{kgs}$

$\overleftrightarrow{\mathcal{F}(\{Z)}=\left\{\mathcal{F}_{1}(0.25), \mathcal{F}_{2}(0.25), \mathcal{F}_{3}(0.5), \mathcal{F}_{4}(0), \mathcal{F}_{5}(0.5)\right\}$

According to this rule Mr. John need walk 15 min, jogging 30 min, running or splits $15 \mathrm{~min}$, yoga and stretching before exercise to keep injury away 30min, and Jim, exercise or any effective sport (graft available on top) $45 \mathrm{~min}$. follow these rules for a week and then increase the intensity mentioned below;

$$
\begin{aligned}
& \mathcal{F}(\{\overleftrightarrow{Y}\})=\left\{\mathcal{F}_{1}(0.25), \mathcal{F}_{2}(0.25), \mathcal{F}_{3}(0.5), \mathcal{F}_{4}(0), \mathcal{F}_{5}(0.5)\right\} \\
& \mathcal{F}(\overleftrightarrow{\{X}\})=\left\{\mathcal{F}_{1}(0.25), \mathcal{F}_{2}(0.25), \mathcal{F}_{3}(0.5), \mathcal{F}_{4}(0), \mathcal{F}_{5}(0.5)\right\} \\
& \mathcal{F}(\{W\})=\left\{\mathcal{F}_{1}(0.25), \mathcal{F}_{2}(0.25), \mathcal{F}_{3}(0.5), \mathcal{F}_{4}(0), \mathcal{F}_{5}(0.5)\right\} \\
& \mathcal{F}(\{V\})=\left\{\mathcal{F}_{1}(0.25), \mathcal{F}_{2}(0.25), \mathcal{F}_{3}(0.5), \mathcal{F}_{4}(0), \mathcal{F}_{5}(0.5)\right\} \\
& \overrightarrow{\mathcal{F}(\{U\})}=\left\{\mathcal{F}_{1}(0.25), \mathcal{F}_{2}(0.25), \mathcal{F}_{3}(0.25), \mathcal{F}_{4}(0.25), \mathcal{F}_{5}(0.5)\right\} \\
& \overrightarrow{\mathcal{F}}(\{T\})=\left\{\mathcal{F}_{1}(0.25), \mathcal{F}_{2}(0.25), \mathcal{F}_{3}(0.25), \mathcal{F}_{4}(0.25), \mathcal{F}_{5}(0.5)\right\}
\end{aligned}
$$

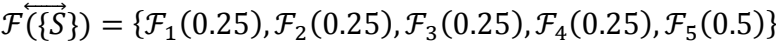

$$
\begin{aligned}
& \overleftrightarrow{\mathcal{F}(\{R}\})=\left\{\mathcal{F}_{1}(0.25), \mathcal{F}_{2}(0.25), \mathcal{F}_{3}(0.25), \mathcal{F}_{4}(0.25), \mathcal{F}_{5}(0.5)\right\} \\
& \widetilde{\mathcal{F}(\{Q\})}=\left\{\mathcal{F}_{1}(0.25), \mathcal{F}_{2}(0.25), \mathcal{F}_{3}(0.25), \mathcal{F}_{4}(0.25), \mathcal{F}_{5}(0.5)\right\} \\
& \mathcal{F}(\overleftrightarrow{\{P}\})=\left\{\mathcal{F}_{1}(0.25), \mathcal{F}_{2}(0.25), \mathcal{F}_{3}(0.25), \mathcal{F}_{4}(0.25), \mathcal{F}_{5}(0.5)\right\} \\
& \mathcal{F}(\{0\})=\left\{\mathcal{F}_{1}(0.25), \mathcal{F}_{2}(0.25), \mathcal{F}_{3}(0.25), \mathcal{F}_{4}(0.25), \mathcal{F}_{5}(0.5)\right\} \\
& \mathcal{F}(\overleftrightarrow{\{N}\})=\left\{\mathcal{F}_{1}(0.25), \mathcal{F}_{2}(0.25), \mathcal{F}_{3}(0.25), \mathcal{F}_{4}(0.25), \mathcal{F}_{5}(0.5)\right\} \\
& \widetilde{\mathcal{F}(\{M}\})=\left\{\mathcal{F}_{1}(0.25), \mathcal{F}_{2}(0.25), \mathcal{F}_{3}(0.25), \mathcal{F}_{4}(0.25), \mathcal{F}_{5}(0.5)\right\} \\
& \overrightarrow{\mathcal{F}(\{L}\})=\left\{\mathcal{F}_{1}(0.25), \mathcal{F}_{2}(0), \mathcal{F}_{3}(0.5), \mathcal{F}_{4}(0.25), \mathcal{F}_{5}(0.5)\right\} \\
& \mathcal{F}(\overleftrightarrow{\{K\}})=\left\{\mathcal{F}_{1}(0.25), \mathcal{F}_{2}(0), \mathcal{F}_{3}(0.5), \mathcal{F}_{4}(0.25), \mathcal{F}_{5}(0.5)\right\} \\
& \mathcal{F}(\overleftrightarrow{U J}\})=\left\{\mathcal{F}_{1}(0.25), \mathcal{F}_{2}(0), \mathcal{F}_{3}(0.5), \mathcal{F}_{4}(0.25), \mathcal{F}_{5}(0.5)\right\} \\
& \mathcal{F}(\{\overleftrightarrow{I}\})=\left\{\mathcal{F}_{1}(0.25), \mathcal{F}_{2}(0), \mathcal{F}_{3}(0.5), \mathcal{F}_{4}(0.25), \mathcal{F}_{5}(0.5)\right\}
\end{aligned}
$$




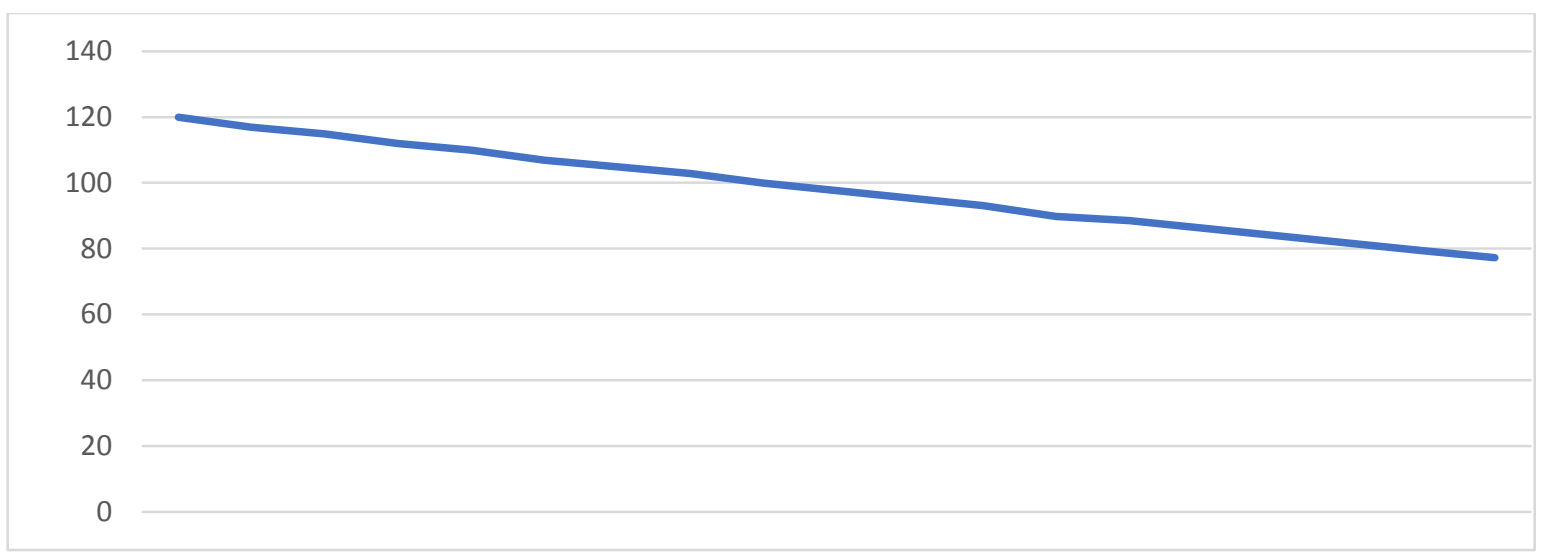

Figure.02

$18 * 7=126$ Days. Hence by multiplying these rules by 7 we get the value of time required for this progress.

\section{CONCLUSION}

In this article, we gave a complete guide to estimate the healthy weight by height, as well as the time duration of exercise and the total time required to get the healthy weight. The healthy weight limit vary with time, by using these different aspects and concept of Fuzzy soft set, we design a system which gave a complete guide to achieve healthy weight loss.

\section{REFERENCES}

[1] G. R. Jahanshahloo, F.H.Lptfi, and M. Izadi Khan.(2006).'An algorithmic method to ext4end TOPSIS for decision-making problems with interval data," Applied Mathematics and Computation, Vol.175, pp. $1375-1384$.

[2] G. W. Wei. (2011). "FIOWHM operator and its application to multiple attribute group decision making," Expert System with Applications, vol. 38, no. 4, pp. 2984-2989.

[3] G. W. Wei, X. F. Zhao, R. Lin and H. J. Wang. (2010). 'Generalized triangular fuzzy correlated averaging operator and their application to multiple attribute decision making," Applied Mathematical Modelling, vol. 36, no. 7, pp. 2975-2982.

[4] Jafar.N.M, Faizullah, Shabbir.S, Alvi.F.M.S, Shaheen.L (2020), Intuitionistic Fuzzy Soft Matrices, Compliments and Their Relations with Comprehensive Study of Medical Diagnosis, International Journal of Latest Engineering Research and Applications, 5(1): 23-30.

[5] Jafar.N.M, Khan.R.M, Sultan.H, Ahmad.N (2020), Interval Valued Fuzzy Soft Sets and Algorithm of IVFSS Applied to the Risk Analysis of Prostate Cancer, International Journal of Computer Applications, 177(38): 18-26.

[6] Jafar.N.M, Muniba.K, Saeed.A, Abbas.S,Bibi.I (2019). Applications of Sanchez, Approach in Medical Diagnosis using Trapezoidal Fuzzy Numbers. International Journal of Latest Engineering, Research and its Applications .4 (9): 5157.

[7] Jafar.N.M, Saeed.A, Waheed.M, Shafiq.A (2020), A Comprehensive Study of Intuitionistic Fuzzy Soft Matrices and its Applications in Selection of Laptop by Using Score Function, International Journal of Computer Applications, 177(38): 8-17.
[8] Jafar.N.M, Saqlain.M, Saeed.M, Abbas.Q (2020), Application of Soft-Set Relations and Soft Matrices in Medical Diagnosis using Sanchez's Approach , International Journal of Computer Applications, 177(32): 7-11.

[9] Molodtsov D. Soft set theory-first result. Computers and Mathematics with Applications. 1999; 37:19-31.

[10] Maji PK, Biswas R, Roy AR. Fuzzy soft set. The Journal of Fuzzy Mathematics. 2001; 9(3):677-692. [10]

[11] Pawlak Z. Rough sets. Int. J. Inf. Comp. Sci. 1982; 11:341356.

[12] Pawlak Z. Rough classification, International Journal of Man Machine Studies 20(5), 469-483, 1984.

[13] Q. W. Cao and J. Wu. (2011). " The Extended COWG operators and their application to multiple attribute group decision making problems and interval numbers," Applied Mathematics Modelling, vol. 35, no. 5, pp. 20752086.

[14] Riaz.M, Saeed.M, Saqlain.M, Jafar.N (2019), Impact of Water Hardness in Instinctive Laundry System Based on Fuzzy Logic Controller, Punjab University Journal of Mathematics, 51(4):73-84.

[15] Smarandache, F. A unifying field in logics neutrosophy: Neutrosophic probability, set and logic. American Research Press, Rehoboth. 1998.

[16] Smarandache F., Definition of Neutrosophic logic-a generalization of the Intuitionistic fuzzy logic, EUSFLAT Conf., 141-146, 2003.

[17] Smarandache F. Neutrosophy and Neutrosophic logic. First International Conference onNeutrosophic Logic, Set, Probability and Statistics. University of New Mexico, Gallup, USA; 2002.

[18] Saqlain.M, Jafar.N, Hamid.R,Shahzad.A. (2019), Prediction of Cricket World Cup 2019 by TOPSIS Technique of MCDM-A Mathematical Analysis, International Journal of Scientific \& Engineering Research, 10(2): 789-792.

[19] Saqlain. M., Jafar. N. and Riffat. A., (2019). Smart phone selection by consumers' in Pakistan: FMCGDM fuzzy multiple criteria group decision making approach, Gomal University Journal of Research, 34(1): 27-31. 
[20] Saqlain.M, Naz.K, Ghaffar.K, Jafar.N.M (2019), Fuzzy Logic Controller: The Impact of Water $\mathrm{pH}$ on Detergents, Scientific Inquiry of Review 3(3):16-29.

[21] Saqlain M, Saeed M, Ahmad M.R, SmarandacheF, (2019), Generalization of TOPSIS for Neutrosophic Hypersoft set using Accuracy Function and its Application, Neutrosophic Sets and Systems (NSS), 27: 131-137.

[22] Saeed, M., Zulqarnain, M. and Dayan, F. (2018). TOPSIS analysis for the prediction of diabetes based on general characteristics of humans. Int. J. of Pharm. Sci. and Research. 9: 2932-2939.

[23] Y. C. Tang and C. T. Chang. (2012). 'Multi-criteria decision-making based on goal programing and fuzzy analytic hierarchy process: an application to capital budgeting problem," Knowledge-Based System, vol. 26, pp. 288-293.

[24] Zadeh L.A..Fuzzy sets as a basis for a theory of possibility, Fuzzy sets and system1 (1), 3-28, 1978.

[25] Zadeh L.A. Fuzzy sets. Inf. Control. 1965; 8:338-353. 